\title{
Finite dimensional interconnections and stabilization of 2D behaviors
}

\author{
Diego Napp and Paula Rocha
}

\begin{abstract}
This paper deals with discrete two-dimensional behaviors which are described by linear systems of partial difference equations with constant coefficients. Within the behavioral framework a natural concept of interconnection has been introduced by J.C.Willems, called regular interconnection. We investigate regular interconnections that yield finite dimensional behaviors, and prove that when a finite dimensional behavior can be achieved from a given behavior $\mathfrak{B}$ by regular interconnection then the controllable part of $\mathfrak{B}$ is rectifiable. We apply this result to characterize all stabilizable behaviors.
\end{abstract}

\section{INTRODUCTION}

As is well known, the central idea in the behavioral approach to control is the one of interconnection. This consists in the interconnection of a given behavior to be controlled $\mathfrak{B}$ (the plant) with a suitable behavior (the controller), in order to obtain a desired behavior $\mathfrak{B}^{d}$. If this is possible, we say that $\mathfrak{B}^{d}$ is implementable from $\mathfrak{B}$.

In this paper we focus on a particular kind of interconnection that is called regular interconnection. In such interconnection, the restrictions imposed on the plant by the controller are independent of the restrictions already present in the plant, as happens, for instance, in a feedback interconnection (see [6]).

More concretely, we are interested in studying regular interconnections that yield finite dimensional behaviors, i.e., we wish to characterize the behaviors from which a finite dimensional behavior is implementable by regular interconnection.

This can be seen as a relaxation of the control objective of implementing the zero behavior by regular interconnection from a given behavior $\mathfrak{B}$, a problem that has already been addressed in [8]. In this sense, regular implementability of a finite dimensional behavior can be regarded as almost regular implementability of zero.

On the other hand our problem is related with the stabilization of 2D behaviors. Indeed the classical stabilization problem can be reformulated in terms of interconnections as the search for a controller behavior whose interconnection with the given behavior yields a stable one. In this context, a behavior that admits a stabilizing controller is said to be stabilizable.

Using a notion of stability defined with respect to a specified stability region by adapting the ideas in [5] to the discrete case, it was recently proven, in [7], that the stable behaviors considered there have the property of being finite dimensional linear subspaces of $\left(\mathbb{R}^{q}\right)^{\mathbb{Z}^{2}}$. Thus, the possibility of stabilizing a behavior $\mathfrak{B}$ is strictly connected with the

Department of Mathematics, University of Aveiro, 3810-193 Aveiro, Portugal.procha@ua.pt,diego@ua.pt regular implementation of a finite dimensional behavior from $\mathfrak{B}$.

A complete characterization of the stabilization property was given in [7] under the assumption that the controllable part of the given behavior $\mathfrak{B}$, denoted by $\mathfrak{B}^{c}$, is rectifiable, i.e., is a direct summand of $\left(\mathbb{R}^{q}\right)^{\mathbb{Z}^{2}}$. This is a very strong property and allows to derive several results that are in general only valid for the one dimensional case (1D), such as, for instance the existence of a decomposition of the behavior into the direct sum of its controllable part and an autonomous part.

However, in this paper we prove that if a finite dimensional behavior is implementable by regular interconnection from a given behavior $\mathfrak{B}$, then $\mathfrak{B}^{c}$ is rectifiable. As a consequence of this result we conclude that the assumption about the rectifiability of $\mathfrak{B}^{c}$, used in [8] in order to obtain several results on stabilization, is indeed not restrictive since it is a necessary condition for stabilization.

The outline of the paper is as follows. We begin by introducing some necessary background from the field of 2D discrete behavioral theory. Most of this material is standard, centering around concepts such as autonomy, controllability and rectifiability. Section 3 is devoted to an exposition of regular interconnection and finite dimensional behaviors. Finally, in Section 4 we introduce the notions of stable and stabilizable behavior and obtain a characterization of all stabilizable behaviors.

\section{Preliminaries}

In order to state more precisely the questions to be considered, we introduce some preliminary notions and results.

We consider $2 \mathrm{D}$ behaviors $\mathfrak{B}$ defined over $\mathbb{Z}^{2}$ that can be described by a set of linear partial difference equations, i.e.,

$$
\mathfrak{B}=\operatorname{ker} R\left(\underline{\sigma}, \underline{\sigma}^{-1}\right):=\left\{w \in \mathcal{U} \mid R\left(\underline{\sigma}, \underline{\sigma}^{-1}\right) w \equiv 0\right\},
$$

where $\mathcal{U}$ is the trajectory universe, here taken to be $\left(\mathbb{R}^{q}\right)^{\mathbb{Z}^{2}}$, $\underline{\sigma}=\left(\sigma_{1}, \sigma_{2}\right), \underline{\sigma}^{-1}=\left(\sigma_{1}^{-1}, \sigma_{2}^{-1}\right)$, the $\sigma_{i}$ 's are the elementary 2D shift operators (defined by $\sigma_{i} w(\underline{k})=w\left(\underline{k}+e_{i}\right.$ ), for $\underline{k} \in \mathbb{Z}^{2}$, where $e_{i}$ is the $i$ th element of the canonical basis of $\left.\mathbb{Z}^{2}\right)$ and $R\left(\underline{s}, \underline{s}^{-1}\right)$ is a 2D Laurent-polynomial matrix known as representation of $\mathfrak{B}$. Since there is no ambiguity, we will sometimes drop the reference to the variables $\left(\underline{s}, \underline{s}^{-1}\right)$ and write, for instance, $R$ for $R\left(\underline{s}, \underline{s}^{-1}\right)$.

Instead of characterizing $\mathfrak{B}$ by means of a representation matrix $R$, it is also possible to characterize it by means of its orthogonal module $\operatorname{Mod}(\mathfrak{B})$, which consists of all the 2D Laurent-polynomial rows $r\left(\underline{s}, \underline{s}^{-1}\right) \in \mathbb{R}^{1 \times q}\left[\underline{s}, \underline{s}^{-1}\right]$ such that $\mathfrak{B} \subset \operatorname{ker} r\left(\underline{\sigma}, \underline{\sigma}^{-1}\right)$, and can be shown to coincide with 
the $\mathbb{R}\left[\underline{s}, \underline{s}^{-1}\right]$-module $\mathrm{RM}(R)$ generated by the rows of $R$, i.e., $\operatorname{Mod}(\mathfrak{B})=\operatorname{RM}\left(R\left(\underline{s}, \underline{s}^{-1}\right)\right)$ and therefore there is a one-to-one correspondence between $\mathfrak{B}$ and $\operatorname{Mod}(\mathfrak{B})$.

It turns out that sums and intersections of behaviors can be formulated in terms of the corresponding modules.

Theorem 1: [13, pag.1074] Let $\mathfrak{B}^{1}$ and $\mathfrak{B}^{2}$ be two 2D behaviors. Then, $\mathfrak{B}^{1}+\mathfrak{B}^{2}$ and $\mathfrak{B}^{1} \cap \mathfrak{B}_{2}$ are also 2D behaviors and

1) $\operatorname{Mod}\left(\mathfrak{B}^{1}+\mathfrak{B}^{2}\right)=\operatorname{Mod}\left(\mathfrak{B}^{1}\right) \cap \operatorname{Mod}\left(\mathfrak{B}^{2}\right)$

2) $\operatorname{Mod}\left(\mathfrak{B}^{1} \cap \mathfrak{B}^{2}\right)=\operatorname{Mod}\left(\mathfrak{B}^{1}\right)+\operatorname{Mod}\left(\mathfrak{B}^{2}\right)$

The notions of controllability and autonomy play an important role in the sequel.

Definition 2: A behavior $\mathfrak{B} \subset\left(\mathbb{R}^{q}\right)^{\mathbb{Z}^{2}}$ is said to be controllable if for all $w_{1}, w_{2} \in \mathfrak{B}$ there exits $\delta>0$ such that for all subsets $U_{1}, U_{2} \subset \mathbb{Z}^{2}$ with $d\left(U_{1}, U_{2}\right)>\delta$, there exists a $w \in \mathfrak{B}$ such that $\left.w\right|_{U_{1}}=\left.w_{1}\right|_{U_{1}}$ and $\left.w\right|_{U_{2}}=\left.w_{2}\right|_{U_{2}}$.

It was shown (see [10]) that this is equivalently to say that $\mathbb{R}^{1 \times q}\left[\underline{s}, \underline{s}^{-1}\right] / \operatorname{Mod}(\mathfrak{B})$ is a torsion free $\mathbb{R}\left[\underline{s}, \underline{s}^{-1}\right]$-module. On the other hand, we say that a behavior is autonomous if it has no free variables (or inputs). $\mathfrak{B}=\operatorname{ker} R\left(\underline{\sigma}, \underline{\sigma}^{-1}\right)$ is autonomous if and only if $R\left(\underline{s}, \underline{s}^{-1}\right)$ has full column rank (over $\mathbb{R}\left[\underline{s}, \underline{s}^{-1}\right]$ ), [11].

In the $1 \mathrm{D}$ case, all autonomous behaviors are finitedimensional vector spaces. For general multidimensional variable behaviors this is no longer true. In fact, an autonomous multidimensional behavior that is finitedimensional is called strongly autonomous in [5].

In order to characterize strong controllability we recall the definition of zero of a Laurent-polynomial matrix.

Given a 2D Laurent-polynomial matrix $R\left(\underline{s}, \underline{s}^{-1}\right)$ with full column rank, a zero of $R$ is defined as $\underline{\lambda} \in(\mathbb{C} \backslash\{0\})^{2}$ such that

$$
\operatorname{rank} R\left(\underline{\lambda}, \underline{\lambda}^{-1}\right)<\operatorname{rank} R\left(\underline{s}, \underline{s}^{-1}\right),
$$

where, the first rank is taken over $\mathbb{C}$ and the second one over $\mathbb{R}\left[\underline{s}, \underline{s}^{-1}\right]$.

Theorem 3: (see [4, Th.17, Th.28] [11, Cor.1 pag. 141]) Let $\mathfrak{B}=\operatorname{ker} R$ be a $2 \mathrm{D}$ behavior. Then the following are equivalent.

1) $\mathfrak{B}$ has finite dimension,

2) $\mathbb{R}^{1 \times q}\left[\underline{s}, \underline{s}^{-1}\right] / \operatorname{Mod}(\mathfrak{B})$ has finite dimension,

3) for all $i=1,2$ there exists a non-zero polynomial $p_{i}\left(\sigma_{i}\right) \in \mathbb{R}\left[s_{i}, s_{i}^{-1}\right]$ such that

$$
p_{i} \cdot\left(\mathbb{R}^{1 \times q}\left[\underline{s}, \underline{s}^{-1}\right] / \operatorname{Mod}(\mathfrak{B})\right)=0,
$$

4) $R$ has full column rank and $\mathfrak{B}$ has a finite number of zeros.

As also shown in [11], every $2 \mathrm{D}$ behavior $\mathfrak{B}$ can be decomposed into a sum

$$
\mathfrak{B}=\mathfrak{B}^{c}+\mathfrak{B}^{a},
$$

where $\mathfrak{B}^{c}$ is the controllable part of $\mathfrak{B}$ (defined as the largest controllable sub-behavior of $\mathfrak{B}$ ) and $\mathfrak{B}^{a}$ is a (non-unique) autonomous sub-behavior said to be an autonomous part of $\mathfrak{B}$.

Remark 4: It is important to remark that if $\mathfrak{B}=\operatorname{ker} R$ and $\mathfrak{B}^{c}=\operatorname{ker} R^{c}$ then the rank of $R$ and the rank of $R^{c}$ must coincide. Indeed, by [3, Th. 2.69] the number of free variables (inputs) in a behavior $\mathfrak{B}=\operatorname{ker} R \in\left(\mathbb{R}^{q}\right)^{\mathbb{Z}^{2}}$ is given by $q$-rank $R$. On the other hand by [8, Cor. 2.10], $\mathfrak{B}$ and $\mathfrak{B}^{c}$ must have the same number of inputs.

If the controllable-autonomous decomposition happens to be a direct sum decomposition, i.e., if $\mathfrak{B}=\mathfrak{B}^{c} \oplus \mathfrak{B}^{a}$, we say that the autonomous part of $\mathfrak{B}^{a}$ is an autonomous direct summand of $\mathfrak{B}$.

An interesting case is when the controllable part $\mathfrak{B}^{c}$ is rectifiable. A $2 \mathrm{D}$ behavior $\mathfrak{B}=\operatorname{ker} R\left(\underline{\sigma}, \underline{\sigma}^{-1}\right) \subset\left(\mathbb{R}^{q}\right)^{\mathbb{Z}^{2}}$ is said to be rectifiable if there exists an invertible operator $U\left(\underline{\sigma}, \underline{\sigma}^{-1}\right)$, where $U\left(\underline{s}, \underline{s}^{-1}\right)$ is a 2D Laurent-polynomial matrix, such that

$$
U\left(\underline{\sigma}, \underline{\sigma}^{-1}\right)(\mathfrak{B}):=\{U w \mid w \in \mathfrak{B}\}=\left\{v \mid R U^{-1} v=0\right\}
$$

is equal to $\operatorname{ker}\left[\begin{array}{ll}I_{l} & 0\end{array}\right]$, where $I_{l}$ is the $l \times l$ identity matrix, for some $l \in\{1, \ldots, q\}$.

The following theorem shows several characterizations of rectifiable behaviors that have been appeared in several papers.

Theorem 5: (see [8, Lemma 2.12] and [12, Th. 9 and Th. 10 , page 819]) Let $\mathfrak{B}=\operatorname{ker} R$ be a behavior. Then the following are equivalent.

1) $\mathfrak{B}$ is rectifiable,

2) $R$ is zero left prime (ZLP),

3) $\mathfrak{B}$ is direct summand of $\left(\mathbb{R}^{q}\right)^{\mathbb{Z}^{2}}$,

4) $\mathbb{R}^{1 \times q}\left[\underline{s}, \underline{s}^{-1}\right] / \operatorname{Mod}(\mathfrak{B})$ is free.

When a rectifying operator exists, it is possible to take advantage of the simplified form of the rectified behaviors in order to derive various results. In particular, it is not difficult to obtain the next proposition.

Proposition 6: Let $\mathfrak{B}=\operatorname{ker} R\left(\underline{\sigma}, \underline{\sigma}^{-1}\right) \subset\left(\mathbb{R}^{q}\right)^{\mathbb{Z}^{2}}$ be a $2 \mathrm{D}$ behavior with rectifiable controllable part $\mathfrak{B}^{c}$ and $U\left(\underline{\sigma}, \underline{\sigma}^{-1}\right)$ be a corresponding rectifying operator such that $U\left(\underline{\sigma}, \underline{\sigma}^{-1}\right)\left(\mathfrak{B}^{c}\right)=\operatorname{ker}\left[\begin{array}{ll}I_{l} & 0\end{array}\right]$. Then the following are equivalent.

1) $\mathfrak{B}=\mathfrak{B}^{c} \oplus \mathfrak{B}^{a}$

2) $\mathfrak{B}^{a}=\operatorname{ker}\left(\left[\begin{array}{cc}P & 0 \\ X & I_{q-l}\end{array}\right] U\right)$, with $P\left(\underline{s}, \underline{s}^{-1}\right)$ such that $R U^{-1}=\left[\begin{array}{ll}P & 0\end{array}\right]$ and $X\left(\underline{s}, \underline{s}^{-1}\right)$ an arbitrary Laurent-polynomial matrix of suitable size.

Note that the behaviors $\mathfrak{B}^{a}$ of Proposition 6 always exist and are autonomous. Thus, this result states that every behavior with rectifiable controllable part has autonomous direct summands and, moreover, gives a parametrization for all such summands. This yields the following parametrization 
of $U(\mathfrak{B})$.

Corollary 7: Let $\mathfrak{B}=\operatorname{ker} R\left(\underline{\sigma}, \underline{\sigma}^{-1}\right) \subset\left(\mathbb{R}^{q}\right)^{\mathbb{Z}^{2}}$ be a $2 \mathrm{D}$ behavior with rectifiable controllable part $\mathfrak{B}^{c}$ and $U\left(\underline{\sigma}, \underline{\sigma}^{-1}\right)$ be a corresponding rectifying operator such that $U\left(\underline{\sigma}, \underline{\sigma}^{-1}\right)\left(\mathfrak{B}^{c}\right)=\operatorname{ker}\left[\begin{array}{ll}I_{l} & 0\end{array}\right]$. Then

$$
U(\mathfrak{B})=\left[\begin{array}{c}
0 \\
\left(\mathbb{R}^{q-l}\right)^{\mathbb{Z}^{2}}
\end{array}\right] \oplus\left[\begin{array}{l}
I_{l} \\
Y
\end{array}\right] \operatorname{ker} P,
$$

with $P\left(\underline{s}, \underline{s}^{-1}\right)$ such that $R U^{-1}=\left[\begin{array}{ll}P & 0\end{array}\right]$ and $Y\left(\underline{s}, \underline{s}^{-1}\right)$ an arbitrary Laurent-polynomial matrix of suitable size.

Example 8: Let $\mathfrak{B}$ be a $2 \mathrm{D}$ behavior represented by

$$
\mathfrak{B}=\operatorname{ker}\left(\begin{array}{ccc}
s_{1}-1 & s_{1} s_{2}^{2}-s_{2}^{2}-1+s_{1} & s_{1} s_{2}-s_{2} \\
0 & s_{1}+s_{2} & -s_{1}-s_{2}
\end{array}\right) .
$$

Choose the unimodular matrix

$$
U^{-1}=\left(\begin{array}{ccc}
1 & -s_{2}^{2}-1 & -s_{2}^{2}-s_{2}-1 \\
0 & 1 & 1 \\
0 & 0 & 1
\end{array}\right)
$$

in order to obtain

$$
U\left(\mathfrak{B}^{c}\right)=\operatorname{ker} R^{c} U^{-1}=\operatorname{ker}\left(\begin{array}{lll}
1 & 0 & 0 \\
0 & 1 & 0
\end{array}\right)
$$

and

$$
U(\mathfrak{B})=\left[\begin{array}{c}
0 \\
0 \\
(\mathbb{R})^{\mathbb{Z}^{2}}
\end{array}\right] \oplus\left[\begin{array}{cc}
1 & 0 \\
0 & 1 \\
y_{1} & y_{2}
\end{array}\right] \operatorname{ker}\left(\begin{array}{cc}
s_{1}-1 & 0 \\
0 & s_{1}+s_{2}
\end{array}\right)
$$

where $y_{1}, y_{2}$ are arbitrary Laurent-polynomials.

\section{CONTROL, REGULAR INTERCONNECTIONS AND $\mathfrak{B}^{c}$}

Given two behaviors $\mathfrak{B}^{1}$ and $\mathfrak{B}^{2}$ their interconnection is defined as the intersection $\mathfrak{B}^{1} \cap \mathfrak{B}^{2}$. This interconnection is said to be regular if

$$
\operatorname{Mod}\left(\mathfrak{B}^{1}\right) \cap \operatorname{Mod}\left(\mathfrak{B}^{2}\right)=\{0\} .
$$

Regular interconnections correspond to a lack of overlapping between the laws of the interconnected behaviors and play an important role in behavioral control, [9], [13], [6], [1]. The following result can be found in, for instance, [8, Lemma 3, pag 115].

Lemma 9: Given the two behaviors $\mathfrak{B}^{1}=\operatorname{ker} R^{1}$ and $\mathfrak{B}^{2}=\operatorname{ker} R^{2}$, the following are equivalent.

1) $\mathfrak{B}^{1} \cap \mathfrak{B}^{2}$ is a regular interconnection,

2) $\mathfrak{B}^{1}+\mathfrak{B}^{2}=\left(\mathbb{R}^{q}\right)^{\mathbb{Z}^{2}}$,

3) $\operatorname{rank} R^{1}+\operatorname{rank} R^{2}=\operatorname{rank}\left(\begin{array}{c}R^{1} \\ R^{2}\end{array}\right)$

Thus in a regular interconnection, the controller imposes restrictions which are not already present in the plant. In this sense a feedback controller is a simple example of a regular interconnection where the controller imposes restrictions only on the plant input, which in the plant is unrestricted.

Example 10: Let $\mathfrak{B}^{1}$ a behavior represented by

$$
\mathfrak{B}^{1}=\operatorname{ker}\left(\begin{array}{ccc}
s_{1} & s_{1} s_{2} & 0 \\
s_{1}+s_{2} & 0 & 1
\end{array}\right)
$$

and $\mathfrak{B}^{2}=\operatorname{ker}\left(\begin{array}{lll}1 & 0 & 1\end{array}\right)$ be two behaviors. Then the interconnection of $\mathfrak{B}^{1}$ and $\mathfrak{B}^{2}$ is regular, i.e., $\operatorname{Mod}\left(\mathfrak{B}^{1}\right) \cap$ $\operatorname{Mod}\left(\mathfrak{B}^{2}\right)=\{(0,0,0)\}$.

Based on the notion of behavior interconnection it is possible to formulate a control problem in set theoretic terms. Indeed, if $\mathfrak{B}$ is the behavior of the system to be controlled (the plant) and $\mathcal{K}$ is the set of all signals compatible with the additional restrictions to be imposed on $w$, i.e., the controller, then the resulting controlled behavior is given by the interconnection

$$
\mathfrak{B} \cap \mathcal{K}
$$

of the behaviors $\mathfrak{B}$ and $\mathcal{K}$. Thus, in the behavioral setting, a control problem consists in, given a desired controlled behavior $\mathfrak{B}^{d}$, finding a controller $\mathcal{K}$ such that its interconnection (1) with the plant behavior $\mathfrak{B}$ results in $\mathfrak{B}^{d}$. In case this interconnection is regular, the controller is called a regular controller and the desired behavior $\mathfrak{B}^{d}$ is said to be achievable or implementable by regular interconnection.

The following necessary condition for implementation by regular interconnection has been derived in $[8$, Th. 4.5 , pag 124].

Theorem 11: Let $\mathfrak{B}$ and $\mathfrak{B}^{d}$ be two behaviors. Then if $\mathfrak{B}^{d}$ is implementable by regular interconnection from $\mathfrak{B}$ then $\mathfrak{B}=\mathfrak{B}^{c}+\mathfrak{B}^{d}$.

Based on the result it is possible to show the next Lemma.

Lemma 12: Let $\mathfrak{B}$ and $\mathcal{K}$ be two $2 \mathrm{D}$ behaviors. If the intersection of $\mathfrak{B}$ and $\mathcal{K}$ is regular then is also the interconnection between $\mathfrak{B}^{c}$ and $\mathcal{K}$.

Proof: Let $\mathfrak{B} \cap \mathcal{K}=\mathfrak{B}^{d}$ with regular interconnection, i.e. $\operatorname{Mod}(\mathfrak{B}) \oplus \operatorname{Mod}(\mathcal{K})=\operatorname{Mod}\left(\mathfrak{B}^{d}\right)$. Using Theorem 11 we have that $\mathfrak{B}=\mathfrak{B}^{c}+\mathfrak{B}^{d}$ or equivalently $\operatorname{Mod}(\mathfrak{B})=$ $\operatorname{Mod}\left(\mathfrak{B}^{c}\right) \cap \operatorname{Mod}\left(\mathfrak{B}^{d}\right)=\operatorname{Mod}\left(\mathfrak{B}^{c}\right) \cap(\operatorname{Mod}(\mathfrak{B}) \oplus \operatorname{Mod}(\mathcal{K}))$. Since $\operatorname{Mod}(\mathfrak{B}) \cap \operatorname{Mod}(\mathcal{K})=\{0\}$ and $\operatorname{Mod}\left(\mathfrak{B}^{c}\right) \cap \operatorname{Mod}(\mathcal{K}) \subset$ $\operatorname{Mod}(\mathfrak{B})$ we obtain that $\operatorname{Mod}\left(\mathfrak{B}^{c}\right) \cap \operatorname{Mod}(\mathcal{K})=\{0\}$

Example 13: Let $\mathfrak{B}$ and $\mathcal{K}$ as in Example 10. It is easy to see that

$$
\mathfrak{B}^{c}=\operatorname{ker}\left(\begin{array}{ccc}
1 & s_{2} & 0 \\
s_{1}+s_{2} & 0 & 1
\end{array}\right) .
$$

As expected from Lemma 12 , we have that $\operatorname{Mod}\left(\mathfrak{B}^{c}\right) \cap$ $\operatorname{Mod}(\mathcal{K})=0$ i.e. the interconnection with the controllable part is also regular. 
Lemma 12 shows that the controllable part of a behavior plays an important role in the context of regular interconnections. Since a controller which does not interconnect with $\mathfrak{B}^{c}$ in a regular way, is not a regular controller. Moreover, the following two lemmas, which hold only for the 2D case, will be used to show that the controllable part of $\mathfrak{B}$ must be rectifiable if a strongly controllable (finite dimensional) behavior can be implemented from $\mathfrak{B}$ by regular interconnection.

Lemma 14: (see [2, Th.12]) Let $\mathfrak{B}$ be a behavior. Then there exists a unique (up to isomorphism) free $\mathbb{R}\left[\underline{s}, \underline{s}^{-1}\right]$-module $\operatorname{Mod}(\mathfrak{B})^{+} \subset \mathbb{R}^{1 \times q}\left[\underline{s}, \underline{s}^{-1}\right]$ such that $\operatorname{Mod}(\mathfrak{B})^{+} / \operatorname{Mod}(\mathfrak{B})$ has finite dimension.

Note that $\operatorname{Mod}(\mathfrak{B})^{+}$is the smallest free module containing $\operatorname{Mod}(\mathfrak{B})$ and its computation can be effectively implemented, see [2]

Lemma 15: (see [2, Cor.23]) Let $\mathfrak{B}$ be a behavior and $\mathfrak{B}^{d} \subset \mathfrak{B}$ be a sub-behavior. Then there exists $\mathfrak{B}^{c}$ such that $\left(\mathfrak{B} \cap \mathfrak{B}^{c}\right) / \mathfrak{B}^{d}$ has finite dimension if and only if $\operatorname{Mod}(\mathfrak{B})^{+}$ is direct summand of $\operatorname{Mod}\left(\mathfrak{B}^{d}\right)^{+}$.

Theorem 16: Let $\mathfrak{B}$ be a behavior. If there exists a controller behavior $\mathcal{K}$ such that $\mathfrak{B}^{f d}=\mathfrak{B} \cap \mathcal{K}$ is finite dimensional (strongly autonomous) and the interconnection is regular then $\mathfrak{B}^{c}$ is rectifiable.

Proof: Applying Lemma 15 with $\mathfrak{B}^{d}=0$ one obtains that $\operatorname{Mod}(\mathfrak{B})^{+}$is direct summand of $\operatorname{Mod}(0)^{+}=\mathbb{R}^{1 \times q}\left[\underline{s}, \underline{s}^{-1}\right]$. Define $\mathfrak{B}^{+}$as the unique behavior such that $\operatorname{Mod}(\mathfrak{B})^{+}=\operatorname{Mod}\left(\mathfrak{B}^{+}\right)$. By Theorem 1, $\mathfrak{B}$ is direct summand of $\mathbb{R}^{1 \times q}\left[\underline{s}, \underline{s}^{-1}\right]$. Thus, by Theorem 5 , $\mathfrak{B}^{+}$is a rectifiable behavior and therefore also controllable.

We have that for the $2 \mathrm{D}$ case $\operatorname{Mod}\left(\mathfrak{B}^{c}\right)$ is free (see [3, Th.7.42]) and since $\operatorname{Mod}(\mathfrak{B})^{+}$is the largest free module containing $\operatorname{Mod}(\mathfrak{B})$ we have that $\mathfrak{B}^{c} \subset \overline{\mathfrak{B}} \subset \mathfrak{B}$. Further $\mathfrak{B}^{c}$ is the largest controllable sub-behavior which implies $\mathfrak{B}^{+} \subset \mathfrak{B}^{c}$ and therefore $\mathfrak{B}^{c}=\mathfrak{B}^{+}$is rectifiable.

Remark 17: Note that, according to [8], rectifiability is equivalent to the possibility of obtaining the zero behavior by regular interconnection. Therefore, the possibility of obtaining a finite dimensional behavior by regular interconnection from $\mathfrak{B}$ can be regarded as almost regular interconnection to zero, since it represents the implementation of the zero behavior up to a finite dimensional one. Equivalently, by Theorem 16 and Corollary 7, the regular implementation of a finite dimensional behavior can also be seen as almost rectificability since $\mathfrak{B}$ can be written as $\mathfrak{B}=\mathfrak{B}^{c} \oplus \mathfrak{B}^{f d}$ where $\mathfrak{B}^{f d}$ is a finite dimensional behavior and $\mathfrak{B}^{c}$ is rectifiable, i.e., $\mathfrak{B}$ coincides with a rectifiable behavior up to a finite dimensional one.

\section{Stability and Stabilizability}

A discrete $1 \mathrm{D}$ behavior $\mathfrak{B} \subset\left(\mathbb{R}^{q}\right)^{\mathbb{Z}}$ is said to be stable if all its trajectories tend to the origin as time goes to infinity.
In the $2 \mathrm{D}$ case, we shall define stability with respect to a specified stability region, as in [7] by adapting the ideas in [5] to the discrete case. For this purpose we identify a direction in $\mathbb{Z}^{2}$ with an element $\underline{d}=\left(d_{1}, d_{2}\right) \in \mathbb{Z}^{2}$ whose components are coprime integers, and define a stability cone in $\mathbb{Z}^{2}$ as the set of all positive integer linear combinations of 2 linearly independent directions.

Given a stability cone $S \subset \mathbb{Z}^{2}$, a trajectory $w \in\left(\mathbb{R}^{q}\right)^{\mathbb{Z}^{2}}$ is said to be $S$-stable if it tends to zero along every half line in $S$.

By a half-line associated with a direction $\underline{d} \in \mathbb{Z}^{2}$ we mean the set of all points of the form $\alpha \underline{d}$ where $\alpha$ is a nonnegative integer; clearly, the half-lines in a stability cone $S$ are the ones associated with the directions $\underline{d} \in S$.

A behavior $\mathfrak{B}$ is $S$-stable if all its trajectories are $S$-stable. It turns out that stable behaviors on $\left(\mathbb{R}^{p}\right)^{\mathbb{Z}^{2}}$ must be finite dimensional.

Lemma 18: ([7, Lemma 2]) Every 2D behavior $\mathfrak{B} \subset$ $\left(\mathbb{R}^{q}\right)^{\mathbb{Z}^{2}}$ which is stable with respect to some stability cone $S$ is a finite dimensional linear subspace of the trajectory universe, $\left(\mathbb{R}^{q}\right)^{\mathbb{Z}^{2}}$.

In order to characterize stability, we introduce some preliminary notation. Given two elements $\underline{\lambda}=\left(\lambda_{1}, \lambda_{2}\right) \in \mathbb{C}^{2}$ and $\underline{k}=\left(k_{1}, k_{2}\right) \in \mathbb{Z}^{2}$, we define

$$
\underline{\lambda}^{\underline{k}}:=\lambda_{1}^{k_{1}} \lambda_{2}^{k_{2}} \text {. }
$$

Now, let $\mathfrak{B}$ be an autonomous behavior. Since the set of zeros of the different representations of $\mathfrak{B}$ coincide, we may define the set $\mathcal{N}(\mathfrak{B})$ of zeros of the behavior $\mathfrak{B}$ as the set of zeros of any of its kernel representations. As pointed out in Theorem $3, \mathfrak{B}$ is finite dimensional if and only of $\mathcal{N}(\mathfrak{B})$ is a finite set. In the sequel, whenever we refer to $\mathcal{N}(\mathfrak{B})$ we implicitly suppose that $\mathfrak{B}$ is autonomous.

Theorem 19: ([7, Th. 8]) Let $\mathfrak{B} \subset\left(\mathbb{R}^{q}\right)^{\mathbb{Z}^{2}}$ be a behavior, and let $S$ be a stability cone. The the following are equivalent:

1) $\mathfrak{B}$ is $S$-stable

2) $\mathcal{N}(\mathfrak{B})$ is finite and for every zero $\underline{\lambda} \in \mathcal{N}(\mathfrak{B})$ and every direction $\underline{d} \in S$,

$$
|\underline{\underline{\lambda}}|<1
$$

If we call $\underline{\lambda} \in \mathbb{C}^{2}$ is $S$-stable if for every direction $\underline{d} \in S$, $\left|\underline{\lambda}^{\underline{d}}\right|<1$, Theorem 19 can be rephrased as: $\mathfrak{B}$ is $S$-stable if and only if it has a finite number of zeros and these zeros are $S$-stable.

This is of crucial importance for the study of stability and stabilizability.

As for stabilization, our definition of $S$-stabilizability is similar to the one proposed in [5], but has the extra requirement of regularity.

Definition 20: Given a stability cone $S \subset \mathbb{Z}^{2}$, we say that a behavior $\mathfrak{B} \subset\left(\mathbb{R}^{q}\right)^{\mathbb{Z}^{2}}$ is $S$-stabilizable if there exists an 
$S$-stable sub-behavior $\mathfrak{B}^{s} \subset \mathfrak{B}$ that is achievable from $\mathfrak{B}$ by regular interconnection.

The following theorem provides a characterization of all stabilizable behaviors.

Theorem 21: Let $\mathfrak{B}=\operatorname{ker} R\left(\underline{\sigma}, \underline{\sigma}^{-1}\right) \subset\left(\mathbb{R}^{q}\right)^{\mathbb{Z}^{2}}$ be a behavior and $S \subset \mathbb{Z}^{2}$ be a stability cone. Then the following statements are equivalent.

1) $\mathfrak{B}$ is $S$-stabilizable

2) $\mathfrak{B}^{c}$ is rectifiable and if $U$ is a rectifiable operator such that $R U=\left[\begin{array}{ll}P & 0\end{array}\right]$ then $\operatorname{ker} P\left(\underline{\sigma}, \underline{\sigma}^{-1}\right)$ is $S$-stable.

3) $\mathfrak{B}^{c}$ is rectifiable and every autonomous direct summand of $\mathfrak{B}$ is stable.

Proof: $1 \Rightarrow 2$ : Assume that $\mathfrak{B}$ is $S$-stabilizable. Then, by Lemma 18 and Theorem $16, \mathfrak{B}^{c}$ is rectifiable. If $\mathfrak{B}=$ ker $R=\operatorname{ker} P R^{c}$ with $R^{c}$ such that $\mathfrak{B}^{c}=\operatorname{ker} R^{c}$ and $U$ is a rectifying operator for $\mathfrak{B}^{c}$ then $P R^{c}=P\left(\begin{array}{ll}I & 0\end{array}\right) U$, $U(\mathfrak{B})=\operatorname{ker}(P \quad 0)$ and $U\left(\mathfrak{B}^{c}\right)=\operatorname{ker}\left(\begin{array}{ll}I & 0\end{array}\right)$.

If $\mathcal{K}=\operatorname{ker}\left(K_{1} K_{2}\right) U$ is a controller behavior such that its interconnection with $\mathfrak{B}$ is regular and yields an autonomous behavior then, by Lemma 9,

$$
\operatorname{rank}\left(\begin{array}{cc}
P & 0 \\
K_{1} & K_{2}
\end{array}\right)=\operatorname{rank}\left(\begin{array}{cc}
P & 0
\end{array}\right)+\operatorname{rank}\left(\begin{array}{ll}
K_{1} & K_{2}
\end{array}\right) .
$$

On the other hand, $P$ must have full column rank (by remark 4) as well as $K_{2}$ (otherwise $U(\mathfrak{B} \cap \mathcal{K})=$ $\operatorname{ker}\left(\begin{array}{cc}P & 0 \\ K_{1} & K_{2}\end{array}\right)$ would not be full column rank) and therefore we have that

$$
\operatorname{rank}\left(\begin{array}{cc}
P & 0 \\
K_{1} & K_{2}
\end{array}\right)=\operatorname{rank} P+\operatorname{rank} K_{2} .
$$

Thus rank $K_{2}=\operatorname{rank}\left(K_{1} K_{2}\right)$. In particular this implies that for all $\underline{\lambda} \in \mathbb{C}^{2}$

$$
K_{1}\left(\underline{\lambda}, \underline{\lambda}^{-1}\right)=K_{2}\left(\underline{\lambda}, \underline{\lambda}^{-1}\right) \cdot L_{\underline{\lambda}}
$$

for some matrix $L_{\underline{\lambda}}$ with complex entries.

Assume now that $P$ is not stable. Then $\operatorname{rank} P\left(\underline{\lambda}^{*},\left(\underline{\lambda}^{*}\right)^{-1}\right)<\operatorname{rank} P\left(\underline{\sigma}, \underline{\sigma}^{-1}\right)=: \ell$ for some $\underline{\lambda}^{*}$ such that $\left|\underline{\lambda}^{*} \underline{d}\right| \nless 1$ and some $\underline{d} \in S$.

Hence

$$
\begin{array}{r}
M:=\left(\begin{array}{cc}
P\left(\underline{\lambda}^{*},\left(\underline{\lambda}^{*}\right)^{-1}\right) & 0 \\
K_{1}\left(\underline{\lambda}^{*},\left(\underline{\lambda}^{*}\right)^{-1}\right) & K_{2}\left(\underline{\lambda}^{*},\left(\underline{\lambda}^{*}\right)^{-1}\right)
\end{array}\right) \\
=\left(\begin{array}{cc}
P\left(\underline{\lambda}^{*},\left(\underline{\lambda}^{*}\right)^{-1}\right) & 0 \\
K_{2}\left(\underline{\lambda}^{*},\left(\underline{\lambda}^{*}\right)^{-1}\right) \cdot L_{\underline{\lambda}} & K_{2}\left(\underline{\lambda}^{*},\left(\underline{\lambda}^{*}\right)^{-1}\right)
\end{array}\right) \\
=\left(\begin{array}{cc}
P\left(\underline{\lambda}^{*},\left(\underline{\lambda}^{*}\right)^{-1}\right) & 0 \\
0 & K_{2}\left(\underline{\lambda}^{*},\left(\underline{\lambda}^{*}\right)^{-1}\right)
\end{array}\right)\left(\begin{array}{cc}
I & 0 \\
L_{\underline{\lambda}} & I
\end{array}\right)
\end{array}
$$

and since rank $P\left(\underline{\lambda}^{*},\left(\underline{\lambda}^{*}\right)^{-1}\right)<\ell$ it follows that rank $M<$ $\ell$, implying that $U(\mathfrak{B} \cap \mathcal{K})$ and hence $\mathfrak{B} \cap \mathcal{K}$ is not stable.

In this way, we conclude that if $\mathfrak{B}$ is $S$-stabilizable then $P$ must be stable, i.e., $1 \Rightarrow 2$.
$2 \Rightarrow 1$ : Because we can take $\mathcal{K}$ such that $U(\mathcal{K})=$ $\operatorname{ker}(0 \quad I)$.

$2 \Rightarrow 3$ : According to [7, Proposition 1] the autonomous direct summands $\mathfrak{B}^{*}$ of $\mathfrak{B}$ (in the case $\mathfrak{B}^{c}$ is rectifiable) are such that $U\left(\mathfrak{B}^{*}\right)=\operatorname{ker}\left(\begin{array}{ll}P & 0 \\ X & I\end{array}\right)$ with $P$ as before, and $X$ an arbitrary L-polynomial matrix of suitable size. Thus if $P$ is stable so are all the autonomous direct summands of $\mathfrak{B}$.

$3 \Rightarrow 2$ : Obvious, taking into account the form of the autonomous direct summands of $\mathfrak{B}$.

We conclude this paper giving two easy examples.

Example 22: Let $\mathfrak{B}$ be the behavior considered in Example 8 . Then

$$
U(\mathfrak{B})^{a}=\left[\begin{array}{cc}
1 & 0 \\
0 & 1 \\
y_{1} & y_{2}
\end{array}\right] \operatorname{ker}\left(\begin{array}{cc}
s_{1}-1 & 0 \\
0 & s_{1}+s_{2}
\end{array}\right)
$$

where $y_{1}, y_{2}$ are arbitrary Laurent-polynomials. Since $U(\mathfrak{B})^{a}$ is not finite dimensional then $\mathfrak{B}^{a}$ is not finite dimensional and therefore not stable (with respect to any stability cone). Hence we have that $\mathfrak{B}$ is not stabilizable since not every autonomous direct summand of $\mathfrak{B}$ is stable.

Example 23: Let a behavior $\mathfrak{B}$ be represented by $\mathfrak{B}=$ ker $R$ and $S$ the positive orthant, where

$$
R=\left(\begin{array}{cccc}
0 & 1 & 0 & 0 \\
s_{1} s_{2}^{2} & 0 & 0 & 0 \\
s_{1}-2 & 2 s_{1}-s_{1}^{2} & s_{1}-\frac{1}{2} & 0 \\
0 & s_{1}-s_{2}^{3} & s_{2}-\frac{1}{4} & 0
\end{array}\right)
$$

Clearly, another representation of $\mathfrak{B}$ is

$$
\mathfrak{B}=\operatorname{ker}\left(\begin{array}{cccc}
0 & 1 & 0 & 0 \\
1 & 0 & 0 & 0 \\
0 & 0 & s_{1}-\frac{1}{2} & 0 \\
0 & 0 & s_{2}-\frac{1}{4} & 0
\end{array}\right) \text {. }
$$

Further,

$$
\mathfrak{B}^{c}=\operatorname{ker}\left(\begin{array}{llll}
1 & 0 & 0 & 0 \\
0 & 1 & 0 & 0 \\
0 & 0 & 1 & 0
\end{array}\right)
$$

is rectifiable. Hence, $\mathfrak{B}$ is stabilizable since $\mathfrak{B}=\operatorname{ker}(P 0)$ with

$$
\operatorname{ker} P=\operatorname{ker}\left(\begin{array}{ccc}
0 & 1 & 0 \\
1 & 0 & 0 \\
0 & 0 & s_{1}-\frac{1}{2} \\
0 & 0 & s_{2}-\frac{1}{4}
\end{array}\right)
$$

a stable behavior.

\section{ACKNOWLEDGMENTS}

The authors gratefully acknowledge that this work was supported by Fundação para a Ciência e Tecnologia (FCT). 


\section{REFERENCES}

[1] A.A. Julius, J.C. Willems, M.N. Belur, and H.L. Trentelman. The canonical controllers and regular interconnection. Systems Control Lett., 54(8):787-797, 2005.

[2] D. Napp Avelli. Almost direct sum decomposition and implementation of 2D behaviors. Math. Control Signals Systems, 21(1):1-19, 2009.

[3] U. Oberst. Multidimensional constant linear systems. Acta Appl. Math., 20(1-2):1-175, 1990.

[4] U. Oberst. Finite-dimensional systems of partial differential or difference equations. Adv. in Appl. Math., 17(3):337-356, 1996.

[5] H. Pillai and S. Shankar. A behavioral approach to control of distributed systems. SIAM J. Control Optim., 37(2):388-408, 1998.

[6] P. Rocha. Feedback control of multidimensional behaviors. Systems \& Control Letters, 45:207-215, 2002.

[7] P Rocha. Stabilization of multidimensional behaviors. in Multidimens. Systems Signal Process., 19:pp. 273-286, 2008.

[8] P. Rocha and J. Wood. Trajectory control and interconnection of 1D and $n \mathrm{D}$ systems. SIAM J. Control Optim., 40(1):107-134, 2001.

[9] J.C. Willems. On interconnections, control, and feedback. IEEE Trans. Automat. Control, 42(3):326-339, 1997.

[10] J. Wood, E. Rogers, and D.H. Owens. Controllable and autonomous $n$ D linear systems. Multidimens. Systems Signal Process., 10(1):3369, 1999.

[11] E. Zerz. Primeness of multivariate polynomial matrices. Systems Control Lett., 29(3):139-145, 1996.

[12] E. Zerz. Multidimensional behaviours: an algebraic approach to control theory for PDE. Internat. J. Control, 77(9):812-820, 2004.

[13] E. Zerz and V. Lomadze. A constructive solution to interconnection and decomposition problems with multidimensional behaviors. SIAM J. Control Optim., 40(4):1072-1086, 2001/02. 